\title{
WHICH PARAMETERS CONTROL THE LEG MOVEMENT OF A WALKING INSECT? \\ II. THE START OF THE SWING PHASE
}

\author{
BY H. CRUSE \\ Faculty of Biology, Universität Bielefeld, Postfach 8640, D-4800 Bielefeld, F.R.G.
}

Accepted 26 October 1984

\section{SUMMARY}

When a stick insect (Carausius morosus) walks on a treadwheel with one leg standing on a platform beside the wheel, this leg can be considered to perform a prolonged stance phase. To elicit a swing phase in this situation, both load and position must decline below definite threshold values.

The swing phase can be elicited when - given a sufficiently posterior leg position - a central temporal signal initiates a small forward movement, and this is followed by a decrease of load.

It is possible for signals from the next posterior leg to change the position threshold at which the swing phase can be started, but these commands do not influence the force values during the stance phase.

Thus position is one parameter used for the decision to end the stance phase. But it does not serve as a signal for a position feedback mechanism controlling leg movement during the stance phase.

\section{INTRODUCTION}

The movement of a walking leg can be divided into two parts: stance phase, when the leg is on the ground, and swing phase after the leg has been lifted off the ground. The end of stance phase is generally considered to be the main (if not the only) point at which the movement of one leg is coordinated with that of the other legs (Graham, 1972, 1977; Bässler, 1977; Graham \& Cruse, 1981; Dean \& Wendler, 1982; Cruse \& Epstein, 1982). Thus, it is important to consider which parameters control the end of stance phase.

One parameter used by the central nervous system in controlling leg movement is position. For swing phase, it has been shown that the end is determined by geometrical parameters alone (Cruse, 1979; Dean \& Wendler, 1983; Cruse, Dean \& Suilmann, 1984). For stance phase, Bässler (1977) has demonstrated that the end does not occur when the coxal hairplates and hair rows (position-measuring sense organs) record that the leg is in an extreme anterior position. However, it is not clear 
whether a normal posterior position as such is sufficient to elicit swing movement. Thus, it is unclear whether the actual end of the stance phase is determined by reaching a definite position value; it is also possible that it is terminated when a given load value has been reached. Bässler (1977) has shown that signals of increased load can prevent the start of the swing phase. It was shown in the companion paper to this (Cruse, 1985b) that under experimental conditions, with one leg standing on a fixed platform and the other five legs walking on the treadwheel, load and position are correlated.

Thus even when position appears to be an important parameter, it may be that load is actually the responsible parameter. In this paper, an experiment is presented which makes it possible to separate these two factors. It will be shown that position is one of the parameters which can determine the end of the stance phase.

\section{METHODS}

Adult female stick insects (Carausius morosus) walked on a styrofoam treadwheel (diameter $38.5 \mathrm{~cm}$, breadth $9 \mathrm{~mm}$, moment of inertia $720 \mathrm{gcm}^{2}$, friction $0.8 \mathrm{mN}$ ) while tethered at the thorax. For details, see the preceding paper (Cruse, 1985b). One leg was placed on a platform attached to the micromanipulator with a 'soft' spring steel band which allowed considerable horizontal movement of the platform when loaded (platform displacement of $8 \mathrm{~mm}$ for $10 \mathrm{mN}$ ). By means of the micromanipulator, the standing leg of the walking animal was placed at the desired position relative to the body. The animal was forced to develop about the same force $(5-7 \mathrm{mN})$ in all tested positions by being stimulated to different levels of excitation. Then the platform was suddenly unloaded horizontally in a backward direction by a weight attached to the platform by a thread running over a pulley. This weight $(1 \cdot 7 \mathrm{~g})$ was chosen to be large enough to take the whole load. The platform was thereby moved further backwards by about $4 \mathrm{~mm}$. It was noted whether after this unloading the leg started a swing movement or remained standing on the platform. The unloading was initiated independently of the rhythmic movements of the walking legs.

All position values are given relative to a coordinate parallel to the long axis of the body, with the origin in front of the tip of the head (Bässler, 1972) and positive values in the anterior direction. As the animals differed in length, all values were normalized to a mean body length of $72 \mathrm{~mm}$ (Cruse, 1976).

\section{RESULTS}

Fig. 1A presents the results for the hind leg, showing the percentage of starts of the swing phase following unloading plotted against position. The data show that no liftoff occurs when the leg is unloaded in the anterior part of its normal range of movement. In contrast, the leg nearly always lifts off when it is unloaded in a position behind the normal endpoint of the stance phase ('posterior extreme position', PEP). Corresponding results were obtained for the middle and front legs. The unloading experiment on the front leg was performed in two ways for each animal: (a) with the 
middle leg standing on a separate fixed platform and (b) with the swing phase of the middle leg blocked by a vertical stick (Dean \& Wendler, 1982; Cruse \& Epstein, 1982). In this way the middle leg was held in either prolonged stance phase or prolonged swing phase. The results (Fig. 1B) show that when the swing phase of the
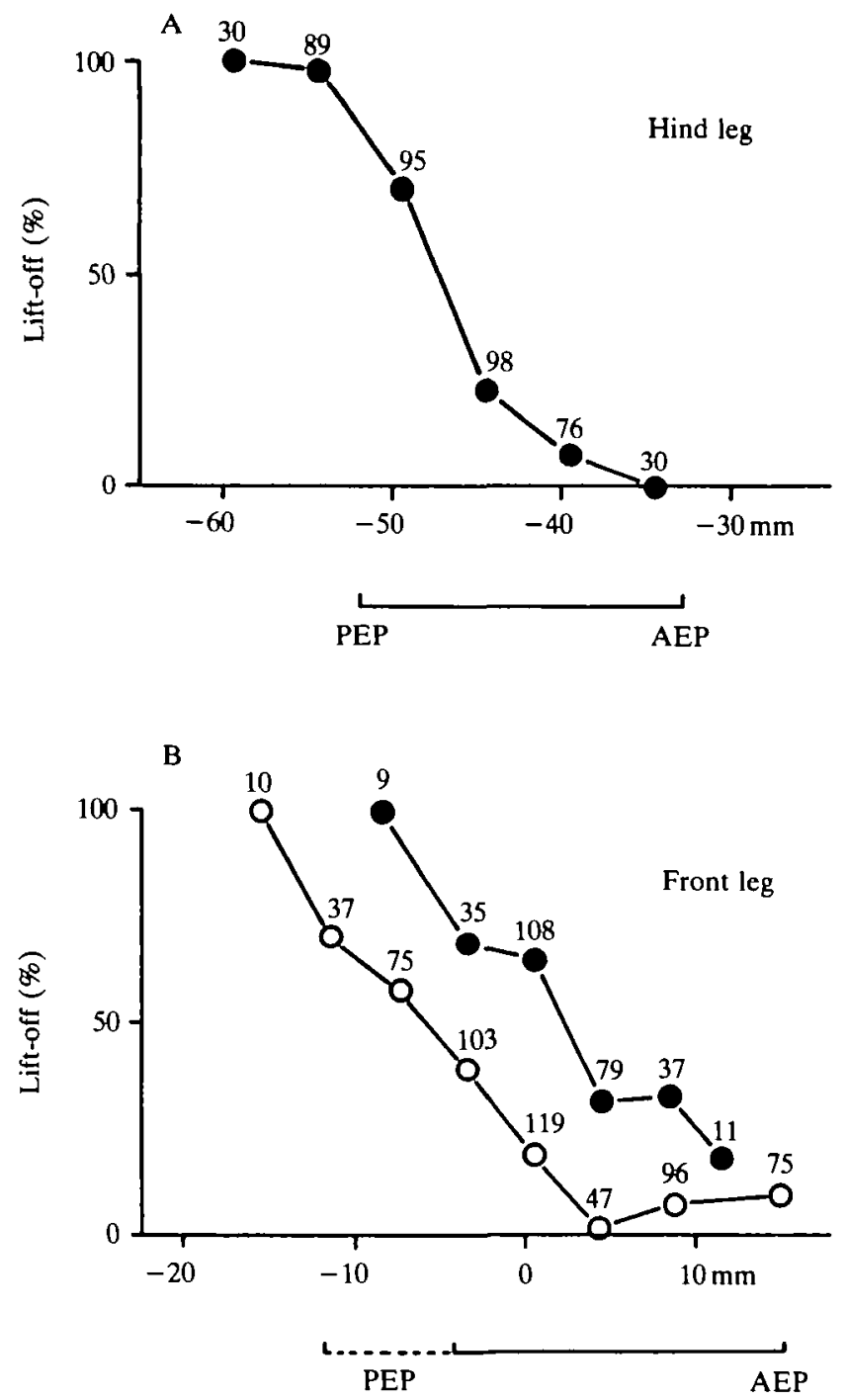

Fig. 1. Percentage of cases in which legs started the swing phase after being unloaded. (A) Hind legs, (B) front legs with the middle leg on a fixed platform (O) or with blocked swing phase (O). Abscissa: position coordinate parallel to the longtudinal axis of the body at the moment before the leg was unloaded. Origin is tip of the head. Positive values are anterior. Below the abscissa the ranges of normal leg movement during walking are given by solid lines. The extension of the range of the front leg when blocking the swing phase of the middle leg is shown by the dashed line. AEP, anterior extreme position; PEP, posterior extreme position. The numbers next to each point show the sample size. Results from four animals. 
middle leg is blocked, the position value at which the swing phase of the front leg is started shifts in a posterior direction.

\section{DISCUSSION}

Previous work (Pearson, 1972; Bässler, 1977) has shown that load is one of the parameters which control the start of the swing phase. The experiments reported here support this finding, as unloading can elicit a swing phase at every point during the cyclic movement of the other walking legs. Indirect evidence from several authors (Bässler, 1979; Graham, 1978; Graham \& Bässler, 1981; Foth \& Graham, 1983; Cruse \& Epstein, 1982, Fig. 2) suggests that pure temporal signals can occur. However, such signals could only be effective if the load of the leg were small enough. The unloading experiment shows that the same applies to the position value. Thus, a load or position value above the threshold can prevent the start of the swing phase even if temporal signals arise. If both values drop below the threshold the swing phase is started immediately, as was shown in the experiment above. The results are supported by recent experiments by Bässler, Foth \& Breutel (1985), who also showed that position information provided by the femoral chordotonal organ can change the state of the front leg from stance to swing.

In all unloading experiments the leg stands on the platform during several steps by the other legs before the experimental stimulus is applied. It is therefore possible that a position signal alone is sufficient to elicit swing phase or that a central command exists which, during a prolonged stance phase, opens a gate to allow the load and the position signals to become effective. Without such a command, the signals from the sense organs alone might not elicit a swing movement. When a leg of a walking animal stands on a platform that leg develops oscillating forces. Bässler (1979) has shown that during the minimum values of such force oscillations the probability increases that a swing phase will start. Therefore it seems unlikely that such a gate remains open during the prolonged stance phase; instead peripheral signals are combined additively with the rhythmic temporal signals, with their sum deciding whether or not the swing phase can be started.

In the preceding paper (Cruse, 1985b, Fig. 2) it was shown that a leg standing on a 'soft' platform (fixed by an elastic steel band to the micromanipulator) remains standing in positions far posterior to those maintained on a stiff platform. The leg moves slightly forward when starting a swing movement. On the stiff platform the leg is immediately unloaded after this small movement, which apparently allows it to continue the swing phase. The soft platform, however, follows the leg. Since the leg is not completely unloaded, further swing movement stops. This can be demonstrated by holding a finger in front of the soft platform to prevent it from moving further forward. After the first small active forward movement on the platform, which is elicited by a central temporal signal, the leg lifts off the soft platform as well. This means that a successful start of the swing phase consists of two parts: a small initial forward movement which must then be followed by a decrease in load. Bässler (1977) 
found similar results: after continuous artificial stimulation of the campaniform sensillae, at the end of the stance phase the leg lifted only the tarsus claws from the ground whilst the other parts of the leg remained standing on the ground. The small negative force values at the end of the stance phase (Cruse, 1985b, Fig. 4) may also reflect this sequence of a central command followed by the sensory feedback necessary to start the swing phase.

When the swing phase of a leg is blocked by a vertical stick, the start of the swing phase of the next anterior leg is delayed and its posterior extreme position is shifted backwards (Dean \& Wendler, 1982; Cruse \& Epstein, 1982). As Fig. 1 shows, this effect is produced not by the delay of a temporal signal but rather by a change of the position threshold.

The results described in the first paper (Cruse, 1985b, Figs 1, 5) indicate that position signals are not used in a feedback loop to control movement during the stance phase. Thus, in summary, position is an important parameter for the decision to finish the stance phase. But it seems not to be used as a parameter for the control of movement during the stance phase. In contrast, load is used for the decision to finish the stance phase and seems to be used as well for control of the leg movement during the stance phase (Pearson, 1972; Bässler, 1977; Cruse, 1985a).

I want to express my thanks to $\mathrm{Dr} D$. Forsythe for correcting the English manuscript. Supported by DFG (Cr 58-4).

\section{REFERENCES}

BXSSLER, U. (1972). Zur Beeinflussung der Bewegungsweise eines Beines von Carausius morosus durch Amputation anderer Beine. Kybemetik 10, 110-114.

BXSSLER, U. (1977). Sense organs in the femur of the stick insect and their relevance to the control of position of the femur-tibia-joint. F. comp. Physiol. 121, 99-113.

BXSSLER, U. (1979). Interactions of central and peripheral mechanisms during walking in first instar stick insects, Extatosoma tiaratum. Physiol. Entomol. 4, 193-199.

BAssler, U., Foth, E. \& BreUtel, G. (1985). The inherent walking direction is different for pro- and metathoracic legs of stick insects. F. exp. Biol. (in press).

Cruse, H. (1976). On the function of the legs in the free-walking stick insect Carausius morosus. 7. comp. Physiol. 112, 235-262.

CRUSE, H. (1979). The control of the anterior extreme position of the hindleg of a walking insect. Physiol. Entomol. 4, 121-124.

CRUSE, H. (1985a). Coactivating influences between neighbouring legs in walking insects. J. exp. Biol. 114, $513-519$.

CruSE, H. (1985b). Which parameters control the leg movement of a walking insect? I. Velocity control during the stance phase. Y. exp. Biol. 116, 343-355.

Cruse, H., Dean, J. \& Sunlmann, M. (1984). The contributions of diverse sense organs in the control of leg movement by a walking insect. F. comp. Physiol. 154, 695-705.

Cruse, H. \& EPSTEIN, S. (1982). Peripheral influences on the movement of the legs in a walking insect Carausius morosus. F. exp. Biol. 101, 161-170.

DEAN, J. \& WENDLER, G. (1982). Stick inBects walking on a wheel: perturbations induced by obstruction of leg protraction. F. comp. Physiol. 148, 195-207.

DEAN, J. \& WENDLER, G. (1983). Stick insect locomotion on a walking wheel: interleg coordination of leg position. 9. exp. Biol. 103, 75-94.

FOTH, E. \& Graham, D. (1983). Influence of loading parallel to the body axis on the walking coordination of an insect. I. Ipsilateral effects. Biol. Cybernetics 47, 17-23.

GraHAM, D. (1972). An analysis of walkung movements in the first instar and adult stick insect Carausius morosus. F. comp. Physiol. 81, 23-52. 
GrAHAM, D. (1977). Simulation of a model for the coordination of leg movement in free walking insects. Biol. Cybernetics 26, 187-198.

Graham, D. (1978). Unusual step patterns in the free walking grasshopper, Neoconocephalus robustus. II. A critical test of the leg interactions underlying different models of hexapod co-ordination $\mathcal{7}$. exp. Biol. 73, 159-172.

GrAHAM, D. \& BASSLER, U. (1981). Effects of afference sign reversal on motor activity in walking stick insects (Carausius morosus). F. exp. Biol. 91, 179-193.

Graham, D. \& Cruse, H. (1981). Coordinated walking of etick insects on a mercury surface. $\mathcal{J}$. exp. Biol. 92, 229-241.

Pearson, K. G. (1972). Central programming and refler control of walking in the cockroach. $\mathcal{F}$. exp. Biol. 56, 173-193. 